\title{
On Unitary Representation Theories of Compact Quantum Groups
}

\author{
Shigeru Yamagami \\ Department of Mathematics, Tohoku University, Sendai, 980, Japan
}

Received: 22 September 1993/in revised form: 10 March 1994

\begin{abstract}
Categorical structure of unitary representations of compact quantum groups is studied with relation to a metrical structure encountered in the monoidal category of bimodules of finite Jones index.
\end{abstract}

\section{Introduction}

It has been long believed that there exists a close relationship between Jones index theory and quantum groups (see [Jo]). One of the striking results is due to $\mathrm{H}$. Wenzl [We1], where a new series of subfactors are constructed via representations of Hecke algebras at roots of unity. The subfactors of this type are further studied in [We2, We3, EK]. All these works are concerned about $q$-deformation of universal enveloping algebras of classical groups with $q$ a root of unity. When the deformation parameter $q$ takes real values, these quantum groups fit into the formalism of compact quantum groups of Woronowicz.

The purpose of the present paper is to clarify the relationship between compact quantum groups and the Jones subfactor theory. More precisely, we shall describe (unitary) Tannaka duals of compact quantum groups in the framework of subfactor theory. To this end we need to adopt the bimodule-approach to Jones index theory developed by A. Ocneanu; given an inclusion relation of factors $N \subset M$, the associated bimodule ${ }_{N} L^{2}(M)_{M}$ (here $L^{2}(M)$ denotes the regular representation of $M)$ and its iterated tensor products determine the whole combinatorial information of the inclusion $N \subset M$.

More generally we can work with monoidal categories which retain the combinatorial structure of bimodules. Indeed, in our previous paper [FRTC], the combinatorial structure of bimodules of the finite Jones index is extracted into a set of categorical axioms: other than rather obvious monoidal structure including conjugation operation, we have encountered a metrical structure arising from minimal expectations, which is called $\varepsilon$-structure there and will be used in the present paper. The monoidal category of finite dimensional unitary representations of a compact group is a typical and important example fulfilling these axioms. So it would be a natural question to ask whether the same structure remains existing for 
compact quantum groups. (Since we will mostly work with the "group algebra" of compact quantum groups, it might be appropriate to call them "discrete quantum groups" as considered in [PW, ER, VD].)

Our main result in the present paper is then to give an affirmative answer to this question.

In that process, we found that compact quantum groups admit a distinguished group-like element inside its group algebra, which is characteristic in the nonunimodularity of compact quantum groups and is directly linked to the $\varepsilon$-structure in the Tannaka duals. Moreover, it turns out to the dualized object of Woronowicz's characters $f_{z}$ constructed for compact matrix pseudogroups. With these reasons, this distinguished element is referred to as a characteristic element in this paper and will play a significant role in the following. Since its existence is easily checked in the known examples, we shall take characteristic elements as ingredients of the compact quantum group. In other words, we shall assume the existence (and a choice) of characteristic elements for compact quantum groups from the outset.

Such an assumption is particularly natural from the viewpoint of subfactor theory and, even in the purely representation-theoretical study of quantum groups, it will deserve much attention. In fact, an explicit use of the characteristic element enables us to define the conjugation of unitary representations in the ideal way and provides the canonical $\varepsilon$-structure in the (unitary) Tannaka duals. Conversely, starting from a monoidal category with $\varepsilon$ structure which is realized as a subcategory of finite-dimensional Hilbert spaces, we can smoothly recover the groupvon Neumann algebra of a compact quantum group, which should be compared with the elaborate construction in ([Wo2]). Furthermore, utilizing the characteristic element, we can easily show the existence and the uniqueness of Plancherel weights for compact quantum groups as well as its explicit formula due to Podles and Woronowicz ([PW, Sect. 3]).

In a subsequent paper, we shall even prove a generalized version of the Hermann-Ocneanu conjecture on a characterization of crossed products in the framework of subfactor theory as a direct application of the present formalism ([CPB, OCCP]).

Other than the fundamental papers [Wo1, Wo2] by Woronowicz, a number of papers are now available in this field. Among them we are indebted to an article by Nakagami $([\mathrm{N}])$, where characteristic elements are dealt with in the form of 1-parameter automorphism groups and it was in fact the starting point of the present work. In the final stage of the present paper, we noticed the works [ER, VD] on discrete quantum groups, where related topics, including the existence and uniqueness problem of invariant weights, are investigated.

The author is grateful to Professors E. Effros, Y. Nakagami, and A. Van Daele for useful information and discussions on the present subject.

Notation and Terminology. A complex vector space $C$ is called a coalgebra if $C$ is furnished with two linear maps $\Delta: C \rightarrow C \otimes C$ and $\varepsilon: C \rightarrow \mathbb{C}$ (called comultiplication and counit respectively) such that $\left(\Delta \otimes 1_{C}\right) \circ \Delta=\left(1_{C} \otimes \Delta\right) \circ \Delta$ and $\left(\varepsilon \otimes 1_{C}\right) \circ \Delta$ $=\mathrm{id}_{C}=\left(1_{C} \otimes \varepsilon\right) \circ \Delta:$ the following diagrams commute.

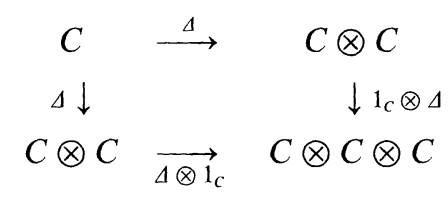


Given a coalgebra $C$, the algebraic dual $C^{\prime}$ of $C$ is in a natural way an algebra: the multiplication in $C^{\prime}$ is given by

$$
v^{\prime} w^{\prime}=\left(v^{\prime} \otimes w^{\prime}\right) \circ \Delta, \quad v^{\prime}, w^{\prime} \in C^{\prime} .
$$

The counit $\varepsilon$ of $C$ is nothing but the unit of this algebra.

An algebra $B$ with unit $1_{B}$ is called a bialbegra if it is a coalgebra at the same time in such a way that

$$
\Delta(a b)=\Delta(a) \Delta(b), \quad \varepsilon(a b)=\varepsilon(a) \varepsilon(b), \quad \Delta\left(1_{B}\right)=1_{B} \otimes 1_{B} .
$$

(Here the multiplication in $B \otimes B$ is given by $\left.(a \otimes b)\left(a^{\prime} \otimes b^{\prime}\right)=\left(a a^{\prime}\right) \otimes\left(b b^{\prime}\right)\right)$.

A bialgebra $(B, \Delta, \varepsilon)$ is called a Hopf algebra if it admits an invertible linear map $\sigma: B \rightarrow B$ satisfying

$$
m \circ\left(\sigma \otimes 1_{B}\right) \circ \Delta=\varepsilon(\cdot) 1_{B}=m \circ\left(1_{B} \otimes \sigma\right) \circ \Delta .
$$

Here $m: B \otimes B \rightarrow B$ denotes the multiplication in $B$.

Such an operator $\sigma$ is, if it exists, unique and called the antinode of the Hopf algebra $(B, \Delta, \varepsilon)$. Antipode is known to be anti-multiplicative and anti-comultiplicative in the following sense (see [Sweedler]):

$$
\begin{gathered}
\sigma(a b)=\sigma(b) \sigma(a), \quad \sigma\left(1_{B}\right)=1_{B}, \\
\sim \circ(\sigma \otimes \sigma) \circ \Delta=\Delta \circ \sigma, \quad \varepsilon \circ \sigma=\varepsilon .
\end{gathered}
$$

Here $\sim: B \otimes B \rightarrow B \otimes B$ denotes the flip map, i.e., $(a \otimes b)^{\sim}=b \otimes a$.

A bialgebra $(B, \Delta, \varepsilon)$ is called a ${ }^{*}$-bialgebra if $B$ is a ${ }^{*}$-algebra with $\Delta$ and $\varepsilon$ preserving the ${ }^{*}$-operation:

$$
\Delta(b)^{*}=\Delta\left(b^{*}\right), \quad \overline{\varepsilon(b)}=\varepsilon\left(b^{*}\right), \quad b \in B,
$$

where the *-operation in $B \otimes B$ is defined by

$$
(a \otimes b)^{*}=a^{*} \otimes b^{*} .
$$

When $(B, \Delta, \varepsilon)$ is a Hopf-algebra with antipode $\sigma$, a $*$-operation is said to satisfy the Woronowicz condition if

$$
\sigma\left(\sigma(b)^{*}\right)=b^{*}, \quad b \in B
$$

A Hopf *-algebra is, by definition, a *-bialgebra with antipode which satisfies the Woronowicz condition.

Here are a few remarks on topology: On occasion it is not easy to deal with tensor products in a topological way. This is particularly the case when one considers $C^{*}$-algebras. On the other hand, for von Neumann algebras, the tensor products are neatly defined and behave well mostly. So a *-bialgebra $(B, \Delta, \varepsilon)$ is called a $\mathbf{W}^{*}$-bialgebra if $B$ is a $\mathbf{W}^{*}$-albegra with $\Delta: B \rightarrow B \otimes B$ and $\varepsilon: B \rightarrow \mathbb{C}$ supposed to be weakly continuous. Here $B \otimes B$ means the tensor product of $\mathrm{W}^{*}$-algebras, which is again a $W^{*}$-algebra.

By a *-category we mean an abelian category such that $\mathrm{Hom}$-sets are vector spaces and they admit *-operation: given a morphism $T: X \rightarrow Y$, we are assigned a new morphism $T^{*}: Y \rightarrow X$ in such a way that (i) $T \rightarrow T^{*}$ is conjugage-linear, (ii) $\left(T^{*}\right)^{*}=T$, and (iii) $(S \circ T)^{*}=T^{*} \circ S^{*}$. If a ${ }^{*}$-category is provided with a 
monoidal structure (i.e., tensor products with unit objects) in a compatible way with the *-structure, then we call it a monoidal *-category. Unit objects in monoidal categories are denoted by $\star$ in the following. A monoidal *-category is said to have conjugation if we are given an assignment $X \Rightarrow X^{*}$ on objects together with a correspondence on morphisms $T: X \rightarrow Y \Rightarrow \bar{T}: X^{*} \rightarrow Y^{*}$ in such a way that $\left(X^{*}\right)^{*}=X, \overline{(\bar{T})}=T$, and $\overline{T^{*}}=(\bar{T})^{*}$ (this new morphism is often denoted by ${ }^{t} T$ and called the transposition of $T$ ). Moreover we assume the existence of coherent (i.e., satisfying hexagonal relations) isomorphisms $(X \otimes Y)^{*} \rightarrow Y^{*} \otimes X^{*}$ under which $(X \otimes Y)^{*}$ and $Y^{*} \otimes X^{*}$ are naturally identified and we do not distinguish them in this paper.

A monoidal *-category with conjugation is simply referred to as a *-monoidal category in the following.

In a ${ }^{*}$-monoidal category $\mathscr{C}$, a family of morphisms $\left\{\varepsilon_{X}: X \otimes X^{*} \rightarrow \star\right\}_{X \in \text { Object }}$ is called an $\varepsilon$-structure if

(i) $\varepsilon_{X \otimes Y}=\varepsilon_{X} \circ\left(1_{X} \otimes \varepsilon_{Y} \otimes 1_{X^{*}}\right)$ (multiplicativity),

(ii) $\varepsilon_{X}\left(T \otimes 1_{X^{*}}\right)=\varepsilon_{Y}\left(1_{Y} \otimes{ }^{t} T\right)$ for $T: Y \rightarrow X$ (centrality),

(iii) $\varepsilon_{X}\left(T \otimes 1_{X^{*}}\right)=0$ iff $T=0$ for $T: Y \rightarrow X$ (faithfullness).

The following is a useful identity which is easily deduced from the above three axioms (see [FRTC, Lemma 2.4]):

$$
\left(1_{X} \otimes \varepsilon_{X^{*}}\right)\left(\varepsilon_{X}^{*} \otimes 1_{X}\right)=1_{X}
$$

An $\varepsilon$-structure is called self-adjoint if $\overline{\varepsilon_{X}}=\varepsilon_{X}$ for any $X$.

When the unit object is irreducible, i.e., $\operatorname{End}(\star)=\mathbb{C} 1$, we can introduce the quantum dimension (or statistical dimension) $d(V) \in \mathbb{R}$ of $V$ by

$$
d(V) 1=\varepsilon_{V} \varepsilon_{V}^{*} .
$$

Quantum dimension takes positive values when *-monoidal categories are related to Hilbert spaces in a suitable way (see [FRTC] for more precise information).

\section{Characteristic Elements}

In this section, we introduce some extra information on the Hopf*-algebra referred to as characteristic elements in the Introduction. Before going into the formal definition, let us begin with some heuristic arguments. Suppose that we are given a Hopf *-algebra $A$ with antipode $\sigma$. Then $\sigma^{2}=\sigma \circ \sigma$ is an automorphism of $A$, i.e., $\sigma^{2}$ is an automorphism with respect to the algebra and coalgebra structure in $A$ at the same time. Taking the square of the Woronowicz condition, we have

$$
* \circ \sigma^{2} \circ *=\sigma^{-2} \text {. }
$$

Here we assume that the automorphism $\sigma^{2}$ admits a suitable square root: we can find an automorphism $\theta$ in $A$ (i.e., $\theta$ preserves multiplication and comultiplication) such that

Put

$$
\sigma^{2}=\theta^{2}, * \circ \theta \circ *=\theta^{-1}, \quad \sigma \theta=\theta \sigma
$$

$$
\tau=\sigma \theta^{-1}=\theta^{-1} \sigma .
$$


Then $\tau$ is an antiautomorphism of $A$, i.e.,

$$
\tau(a b)=\tau(b) \tau(a), \quad \Delta \circ \tau=\sim \circ(\tau \otimes \tau) \circ \Delta
$$

and satisfies $\tau^{2}=\sigma \theta^{-1} \sigma \theta^{-1}=\sigma^{2} \theta^{-2}=\mathrm{id}$, i.e., $\tau$ is an involution. Moreover it commutes with *-operation;

$$
\tau\left(a^{*}\right)=\tau(a)^{*}, \quad a \in A .
$$

This follows from

$$
\begin{aligned}
\tau\left(a^{*}\right) & =\left(\sigma \theta^{-1}\left(a^{*}\right)\right)^{*} \\
& \left.=\sigma^{-1} \sigma\left[\sigma\left(\theta(a)^{*}\right)^{*}\right)\right] \\
& =\sigma^{-1}(\theta(a)) \quad(\text { use Woronowicz condition) } \\
& =\tau(a) \quad\left(\text { use } \tau^{2}=\mathrm{id}\right)
\end{aligned}
$$

Clearly $\tau$ and $\theta$ commute.

Now we further assume that $\theta$ is inner, i.e., there is an invertible element $h$ in $A$ such that $\theta(a)=h a h^{-1}$ for $a \in A$. In this case, looking at the property of $\theta$, it is natural to require that $h$ is hermitian and satisfy

$$
\tau(h)=h^{-1}, \quad \Delta(h)=h \otimes h .
$$

Thus we come to the following definition.

Definition 1.1. Let $A$ be a Hopf *-algebra with antipode $\sigma$. An invertible hermitian element $h$ in $A$ is called a characteristic element if

$$
\Delta(h)=h \otimes h, \quad \sigma(h)=h^{-1}, \quad \sigma^{2}(a)=h^{2} a h^{-2} \quad \text { for } a \in A .
$$

Given a characteristic element $h$, an antiautomorphism $\tau$ is $A$ is defined by

$$
\tau(a)=\sigma\left(h^{-1} a h\right)
$$

and called the transposition.

As will be checked later, Drinfeld-Jimbo's q-deformation of simple Lie algebras with $q \in \mathbb{R}$ especially fits the above definition. Since the characteristic elements in these examples turn out to have representations as unbounded linear operators, it is more convenient to work with the unitarized version:

Definition 1.2. Let $M$ be a von Neumann algebra isomorphic to a direct sum of finite-dimensional matrix algebras. $A W^{*}$-bialgebra $(M, \Delta, \varepsilon)$ is called a compact quantum group-von Neumann algebra if it is furnished with an involutive *-antiautomorphism $\tau$ (called transposition) and a one-parameter (continuous) group of unitaries $\left\{u_{t}\right\}_{t \in \mathbb{R}}$ in $M$ such that

and

$$
\Delta\left(u_{t}\right)=u_{t} \otimes u_{t}, \quad \tau\left(u_{t}\right)=u_{-t}
$$

$$
m\left(1 \otimes\left(\tau \circ \theta_{-i}\right)\right) \circ \Delta(a)=\varepsilon(a) 1_{M}=m\left(\left(\tau \circ \theta_{i}\right) \otimes 1\right) \circ \Delta(a)
$$

for $a \in \mathscr{M}$. Here $\mathscr{M}$ is a dense ${ }^{*}$-subalgebra of $M$ consisting of elements in $M$ with finitely many simple (i.e., factorial) components and $\theta_{-i}$ denotes the analytic continuation of $\theta_{t}=\operatorname{Ad} u_{t}$ to $t=-i$. 
Remark. (i) Let $M \simeq \oplus_{j \geqq 1} M_{j}$ with $M_{j}$ isomorphic to a matrix algebra $M\left(d_{j}, \mathbb{C}\right)$. Then the analytic continuation $\theta_{-i}$ is well-defined on any component $M_{j}$ and the condition about $\tau \circ \theta_{-i}$ should be understood in the following sense: for any $j, k \geqq 1$, denote by $\Delta_{j k}(a)$ the $M_{j} \otimes M_{k}$-component of $\Delta(a)$ in the decomposition $M \otimes M=\oplus_{j, k \geqq 1} M_{j} \otimes M_{k}$. Then we have

$$
\left.m\left(\left(1 \otimes\left(\tau \circ \theta_{-i}\right)\right)\left(\Delta_{j j}(a)\right)\right)=\varepsilon(a) 1_{M_{j}}=m\left(\left(\tau \circ \theta_{-i}\right) \otimes 1\right)\left(\Delta_{j j}(a)\right)\right)
$$

for any $j \geqq 1$.

(ii) Since $\tau\left(u_{t}\right)=u_{-t}, \tau$ and $\theta_{t}$ commute.

(iii) The one-parameter group $\left\{\theta_{t}\right\}$ of automorphisms play a fundamental role in the formulation of duality of quantum groups by Masuda and Nakagami ([MN]). The above definition is therefore a specialized and strengthened version of their formalism to the case of the group-von Neumann algebra of compact quantum groups.

\section{Examples}

Let us begin with a review of definitions on quantum groups introduced in [Drinfeld, Jimbo]. Let $A=\left\{a_{i j}\right\}_{1 \leqq i, j \leqq l}$ be a Cartan matrix with a symmetrizing diagonal matrix $D=\operatorname{diag}\left(d_{1}, \ldots, d_{t}\right): d_{i} \neq 0$ and $d_{i} c_{i j}=d_{j} c_{j i}$ for $1 \leqq i, j \leqq l$.

Let $U_{q}$ be a $\mathbb{C}$-algebra having unit with generators $e_{i}, f_{i}, k_{i}^{ \pm}(1 \leqq i \leqq l)$ and relations

$$
\begin{gathered}
k_{i} k_{i}^{-1}=k_{i}^{-1} k_{i}=1, \quad k_{i} k_{j}=k_{j} k_{i}, \\
k_{i} e_{j} k_{i}^{-1}=q^{d_{i} c_{\imath j}} e_{j}, \quad k_{i} f_{j} k_{i}^{-1}=q^{-d_{i} c_{\imath j}} f_{j}, \\
e_{i} f_{j}-f_{j} e_{i}=\delta_{i j} \frac{k_{i}^{2}-k_{i}^{-2}}{q^{2 d_{i}}-q^{-2 d_{\imath}}}, \\
\sum_{v=0}^{1-c_{\imath_{j}}}(-1)^{v}\left[\begin{array}{c}
1-c_{i j} \\
v
\end{array}\right]_{q^{2 d_{i}}} e_{i}^{1-c_{i j}-v} e_{j} e_{i}^{v}=0(i \neq j), \\
\sum_{v=0}^{1-c_{i j}}(-1)^{v}\left[\begin{array}{c}
1-c_{i j} \\
v
\end{array}\right]_{q^{2 d_{i}}} f_{i}^{1-c_{i j}-v} f_{j} f_{i}^{v}=0(i \neq j) .
\end{gathered}
$$

Here

$$
\begin{aligned}
& {[n] !_{q}=\left(q-q^{-1}\right)\left(q^{2}-q^{-2}\right) \cdots\left(q^{n}-q^{-n}\right)} \\
& {\left[\begin{array}{c}
n \\
m
\end{array}\right]_{q}=\frac{[n] !_{q}}{[m] !_{q}[n-m] !_{q}}}
\end{aligned}
$$

for $0 \leqq m \leqq n$.

The algebra $U_{q}$ is furnished with a Hopf-algebra structure by

$$
\begin{gathered}
\Delta\left(e_{i}\right)=e_{i} k_{i}^{-1}+k_{i} \otimes e_{i}, \Delta\left(f_{i}\right)=f_{i} \otimes k_{i}^{-1}+k_{i} \otimes f_{i}, \Delta\left(k_{i}\right)=k_{i} \otimes k_{i}, \\
\varepsilon\left(e_{i}\right)=\varepsilon\left(f_{i}\right)=0, \quad \varepsilon\left(k_{i}\right)=1, \\
\sigma\left(e_{i}\right)=-q^{-2 d_{i}} e_{i}, \quad \sigma\left(f_{i}\right)=-q^{2 d_{i}} f_{i}, \quad \sigma\left(k_{i}\right)=k_{i}^{-1} .
\end{gathered}
$$


Lemma 2.1. We can define an anti-automorphism $\tau$ (i.e., an anti-multiplicative and anti-comultiplicative linear bijection) of $U_{q}$ by

$$
\tau\left(k_{i}\right)=k_{i}^{-1}, \quad \tau\left(e_{i}\right)=-e_{i}, \quad \tau\left(f_{i}\right)=-f_{i} .
$$

Clearly $\tau$ is involutive and commutes with $\sigma$.

$\because)$ This is checked by showing that $\tau$ preserves the generating relations in the generating ideal. The details are left to the readers.

In the following we assume $q$ is real and introduce a ${ }^{*}$-operation in $U_{q}$ so that

$$
k_{i}^{*}=k_{i}, \quad e_{i}^{*}=f_{i}, \quad f_{i}^{*}=e_{i} .
$$

This is well-defined because the above assignment preserves the generating relations which can be checked by a direct computation (note that $q \in \mathbb{R}$ and $d_{i} c_{i j}=d_{j} c_{j i}$ ). It is also easy to check that this *-operation satisfies the Woronowicz condition. In the following $U_{q}$ is considered to be a Hopf *algebra with this *-operation.

The following can be checked by a direct computation.

Lemma 2.2. Let $\left\{b_{i}\right\}_{1 \leqq i \leqq l} \subset \mathbb{Q}$ be defined by $\sum_{i} b_{i} d_{i} a_{i j}=2 d_{j}$ for $j=1, \ldots, l$ (note that as the Cartan matrix of a semi-simple Lie algebra, $A$ is invertible). Then $b_{i} \in \mathbb{Z}$ for $i=1, \ldots, l$.

The following are also immediate from the definition.

Proposition 2.3. Let $\left\{b_{i}\right\}_{1 \leqq i \leqq l}$ be the sequence of integers in the above lemma and set

$$
h=\prod_{i} k_{i}^{-b_{i}} \in U_{q} .
$$

Then $h$ satisfies the property for the characteristic element for the Hopf ${ }^{*}$-algebra $U_{q}$ : (i) $\sigma^{2}=A d h^{2}$, (ii) $\sigma(h)=h^{-1}$, (iii) $\Delta(h)=h \otimes h$, and (iv) $\tau=\sigma \circ A d h^{-1}=$ $A d h^{-1} \circ \sigma$.

Remark. The above element $h$ coincides with the $q$-deformation of the element in the Cartan subalgebra corresponding to the half sum of positive roots (cf. [R3]).

In the following we use the term compact quantum group to designate a Hopf *-algebra $A$ with a characteristic element which admits sufficiently many finitedimensional unitary representtions: for $a \neq b \in A$, there exists a finite-dimensional unitary representation $\pi$ such that $\pi(a) \neq \pi(b)$ (see the definition at the beginning of Sect. 3).

\section{Unitary Representations}

By a *-representation of a Hopf*-algebra (or a Hopf-von Neumann algebra) $A$, we mean a Hilbert space $V$ together with a left action of $A$ on $V$ which satisfies

$$
(a v \mid w)=\left(v \mid a^{*} w\right), \quad a \in A, \quad v, w \in V .
$$

When $A$ is furnished with a characteristic element $h$, we assume that $h$ is represented by a positive operator. 
Given two *-representations $V$ and $W$, we define their tensor product (representation $V \otimes W$ by

$$
a(v \otimes w)=\Delta(a)(v \otimes w) .
$$

Here the right-hand side is defined by restricting the action of $A \otimes A$ on $V \otimes W$. Since $\Delta$ is a *homomorphism, this gives a *-representation of $A$. The conjugate representation of $V$ is by definition the dual Hilbert space $V^{*}$ ( $=$ the conjugate space of $V$ by the "self-duality" of Hilbert spaces) together with the action of $A$ defined by

$$
a v^{*}=\left(\tau\left(a^{*}\right) v\right)^{*} \quad\left(=v^{*} \tau(a)\right), \quad a \in A .
$$

Here $v^{*} \in V^{*}$ denotes the linear functional on $V$ obtained from $v \in V$ through the inner product. Note that this, in fact, gives a *-representation because $\tau$ commutes with the *-operation.

Suppose that $A$ is furnished with a characteristic element $h$. Then a ${ }^{*}$-representation $V$ of $A$ is called unitary if $h$ is represented by a positive operator in $V$.

Remark. Since $\tau=\sigma^{\circ} A d h$, the conjugate representation $V^{*}$ is, forgetting the *-structure, equivalent to the contra-gradient representation defined by antipode $\sigma([\mathrm{L}, \mathrm{R}, \mathrm{Wo} 1])$.

Proposition 3.1. Let $U, V, W$ be *-representations. Then we have

(i) $(U \otimes V) \otimes W=U \otimes(V \otimes W)$.

(ii) $\left(V^{*}\right)^{*}=V$.

(iii) $(V \otimes W)^{*}=W^{*} \otimes V^{*} \quad\left(\right.$ for $v \in V$ and $w \in W$, the element $(v \otimes w)^{*}$ in $(V \otimes W)^{*}$ is identified with the element $\omega^{*} \otimes v^{*}$ in $\left.W^{*} \otimes \mathrm{V}^{*}\right)$.

$\because$ ) (i) is a consequence of coassociativity of $\Delta$ while (ii) follows from the involutiveness of $\tau$. Property (iii) is checked as follows: Write $\Delta(a)=\sum a_{1} \otimes a_{2}$. Then

$$
\begin{aligned}
a\left(w^{*} \otimes v^{*}\right) & =\Delta(a)\left(w^{*} \otimes v^{*}\right)=\sum a_{1} w^{*} \otimes a_{2} v^{*} \\
& =\sum\left(\tau\left(a_{1}^{*}\right) w\right)^{*} \otimes\left(\tau\left(a_{2}\right)^{*} v\right)^{*} \\
& =\left(\sum \tau\left(a_{2}\right)^{*} v \otimes \tau\left(a_{1}\right)^{*} w\right)^{*} \\
& =\left(\left(\sum \tau\left(a_{2}\right) \otimes \tau\left(a_{1}\right)\right)^{*}(v \otimes w)\right)^{*} \\
& =\left([\sim \circ(\tau \otimes \tau) \circ \Delta(a)]^{*}(v \otimes w)\right)^{*} \\
& =\left(\Delta(\tau(a))^{*}(v \otimes w)\right)^{*} \\
& =\left(\tau(a)^{*}(v \otimes w)\right)^{*} \\
& =a(v \otimes w)^{*} .
\end{aligned}
$$

In accordance with the operations on representations just defined, we can introduce the accompanying operations on intertwiners: if $T: V \rightarrow W$ and $T^{\prime}: V^{\prime} \rightarrow W^{\prime}$ are interwiners, then new intertwiners $T \otimes T^{\prime}: V \otimes V^{\prime} \rightarrow W \otimes W^{\prime}$ and $\bar{T}: V^{*} \rightarrow W^{*}$ are defined by

$$
\left(T \otimes T^{\prime}\right)\left(v \otimes v^{\prime}\right)=(T v) \otimes\left(T^{\prime} v^{\prime}\right), \quad \bar{T} v^{*}=(T v)^{*}
$$

and satisfies the properties required in the *-monoidal category. 
In this way we see that the category of unitary representations of a Hopf *-algebra $A$ with characteristic element has the structure of a *-monoidal category.

From here on we restrict ourselves to finite dimensional *-representations and see how the assumed characteristic element in the Hopf *-algebra defines an $\varepsilon$-structure in a canonical way. Let us begin with some heuristic computations to capture the characteristic element within the monoidal category of *-representations.

Since the unit object is given by the counit $\varepsilon$ if it is regarded as a *-representation of $A$ on $\mathbb{C}$, we need to seek for an element $\xi \in V \otimes V^{*}$ which is invariant under the action of $A: a \xi=\varepsilon(a) \xi$ for $a \in A$.

Now suppose that such $\xi$ is given in the form

$$
\xi=\sum_{j} C v_{j} \otimes v_{j}^{*}
$$

with $C \in \mathscr{B}(V)$ a positive invertible operator and $\left\{v_{j}\right\}$ an orthonormal basis in $V$. Note that $\xi$ does not depend on the choice of an orthonormal basis.

For $a \in A$, write $\Delta(a)=\sum a_{1} \otimes a_{2}$. Then the $A$-invariance of $\xi$ is expressed by

$$
\sum_{j} \sum a_{1} C v_{j} \otimes a_{2} v_{j}^{*}=\varepsilon(a) \sum_{j} C v_{j} \otimes v_{j}^{*} .
$$

Taking partial inner products in the second factor, we see that this relation is equivalent to

$$
\sum_{j} \sum\left(a_{2} v_{j}^{*} \mid v_{i}^{*}\right) a_{i} C v_{j}=\varepsilon(a) C v_{i}, \quad i=1, \ldots, d=\operatorname{dim} V .
$$

Since $\left(v_{i}^{*} \mid a_{2} v_{j}^{*}\right)=\left(v_{i}^{*} \mid\left(\tau\left(a_{2}^{*}\right) v_{j}\right)^{*}\right)=\left(\tau\left(a_{2}^{*}\right) v_{j} \mid v_{i}\right)=\left(\tau\left(a_{2}\right)^{*} v_{j} \mid v_{i}\right)$, this condition is written as

$$
\sum a_{1} C \tau\left(a_{2}\right)=\varepsilon(a) C \text { for } a \in A
$$

with $\Delta(a)=\sum a_{1} \otimes a_{2}$. Comparing this with the relation for the antipode $\sigma=\tau \circ A d h$,

i.e.,

$$
\sum a_{1} \tau\left(h a_{2} h^{-1}\right)=\varepsilon(a) 1
$$

$$
\sum a_{1} h \tau\left(a_{2}\right)=\varepsilon(a) h,
$$

we see that $\xi$ is $A$-invariant if we take $C=h$ on $V$.

Proposition 3.2. Define a linear map $\varepsilon_{V}: V \otimes V^{*} \rightarrow \mathbb{C}$ as the adjoint of

$$
\mathbb{C} \ni z \mapsto z \sum_{j}\left(h v_{j}\right) \otimes v_{j}^{*} \in V \otimes V^{*}
$$

with $\left\{v_{j}\right\}$ an orthonormal basis of $V$. Then $\varepsilon_{V}$ does not depend on the choice of $\left\{v_{j}\right\}$ and gives an intertwiner between $V \otimes \mathrm{V}^{*}$ and $\mathbb{C}_{\varepsilon}$ (i.e., the trivial representation of $A$ given by the counit $\varepsilon$ ).

Theorem 3.3. Let $(A, \Delta, \varepsilon)$ be a Hopf *-algebra with a characteristic element $h$ and $\mathscr{R}$ be the *-monoidal category of finite dimensional *-representations of $A$. Then the family $\left\{\varepsilon_{V}\right\}_{V \in \text { Object }}$ gives a self-adjoint $\varepsilon$-structure in $\mathscr{R}$.

Analogously, a compact quantum group-von Neumann algebra determines the *-monoidal category with $\varepsilon$-structure. 
$\because)$ We need to check that $\left\{\varepsilon_{V}\right\}$ satisfies multiplicativity, centrality, and selfadjointness (the faithfullness is obvious). The multiplicativity is a consequence of $\Delta(h)=h \otimes h:$

$$
\begin{aligned}
\varepsilon_{V}^{*} \otimes W(1) & =\sum_{j, k} h\left(v_{j} \otimes w_{k}\right) \otimes\left(v_{j} \otimes w_{k}\right)^{*} \\
& =\sum_{j, k} h v_{j} \otimes h w_{k} \otimes w_{k}^{*} \otimes v_{j}^{*} \\
& =\left(1_{V} \otimes \varepsilon_{W}^{*} \otimes 1_{V^{*}}\right)\left(\sum_{j} h v_{j} \otimes v_{j}^{*}\right) \\
& =\left(1_{V} \otimes \varepsilon_{W}^{*} \otimes 1_{V^{*}}\right) \varepsilon_{V}^{*}(1) .
\end{aligned}
$$

The centrality can be seen as follows: Identify elements in $W \otimes V^{*}$ with linear maps from $V$ into $W$ in the obvious manner. Then $\left(T \otimes 1_{V^{*}}\right) \varepsilon_{V}^{*}(1)$ is identified with

$$
\left.\sum_{j} \mid T h v_{j}\right)\left(v_{j} \mid=T h_{V}\right.
$$

while $\left(1_{W} \otimes{ }^{t} T\right) \varepsilon_{W}^{*}(1)$ is identified with

$$
\left.\sum_{j} \mid h w_{j}\right)\left(w_{j} \mid T=h_{W} T\right.
$$

Since $T: V \rightarrow W$ is an intertwiner, the above two operators coincide.

The self-adjointness follows from the next lemma.

Lemma 3.4. For any orthonormal basis $\left\{v_{j}\right\}$ in $V$,

$$
\varepsilon_{V}^{*}(1)=\sum_{j}\left(h^{1 / 2} v_{j}\right) \otimes\left(h^{1 / 2} v_{j}\right)^{*}=\sum_{j} v_{j} \otimes\left(h v_{j}\right)^{*} .
$$

$\because$ ) Immediate.

Example 3.5. (cf. [L, R1, R2]). Let $U_{q}$ be the q-deformed Hopf ${ }^{*}$-algebra of a semi-simple Lie algebra. Then finite dimensional irreducible ${ }^{*}$-representations of $U_{q}$ are parametrized by the sequences $m=\left\{m_{i}\right\}_{1 \leqq i \leqq l}$ of non-negative integers: a vector space $0 \neq v_{0}$ in $V$ is called a highest weight vector with highest weight $m$ if $e_{i} v_{0}=0$ and $k_{i} v_{0}=q^{d_{2} m_{\imath}} v_{0}(1 \leqq i \leqq l)$. Then any irreducible finite dimensional ${ }^{*}$-representation $V$ has a highest weight vector with highest weight $m \in \mathbb{Z}_{+}^{l}$ and conversely, for any weight $m \in \mathbb{Z}_{+}^{l}$, there is an irreducible finite dimensional *-representation $V$ with $m$ the highest weight. Note that the fourth root of 1 is excluded because we suppose $k_{i}$ to be positive $(1 \leqq i \leqq l)$.

Lemma 3.6. In the above example, the associated *-manoidal category satisfies the self-duality in [FRTC]: For any irreducible $V$,

$$
\left\|\varepsilon_{V}\right\|=\left\|e_{V^{*}}\right\|
$$

$\because$ ) First note that

$$
\left\|\varepsilon_{V}\right\|^{2}=\left\|\varepsilon_{V}^{*}(1)\right\|^{2}=\left\|\sum_{j} h v_{j} \otimes v_{j}^{*}\right\|^{2}=\operatorname{trace}_{V}\left(h^{2}\right) .
$$


Since $h v^{*}=\left(\tau(h)^{*} v\right)=\left(h^{-1} v\right)^{*}$ for $v \in V$, a similar calculation shows that

$$
\left\|\varepsilon_{V^{*}}\right\|^{2}=\operatorname{trace}_{V}\left(h^{-2}\right) .
$$

Here note that the spectra of $h_{V}$ and $h_{V}^{-1}$ are completely symmetric. This follows from the existence of an element in the Weyl group which just changes the sign of roots up to permutation (see [V] for example) and the fact that the multiplicity of weights in $V$ remains invariant under the action of Weyl group ([R1]). In particular we have $\operatorname{trace}_{V}\left(h^{2}\right)=\operatorname{trace}_{V}\left(h^{-2}\right)$.

Example 3.7. Consider the case $A=(2)$ with $D=(1)$, i.e., $\mathfrak{g}=\mathfrak{s l}(2, \mathbb{C})$. The parametrizing weight set is just $\mathbb{Z}_{+}=\{0,1,2, \ldots\}$ and, for $m \in \mathbb{Z}_{+}, h=k$ is represented on the associated representation space $V$ by the diagonal matrix

$$
h_{V}=\left(\begin{array}{cccc}
q^{m} & & & \\
& q^{m-2} & & \\
& & \ddots & \\
& & & q^{-m}
\end{array}\right) \text {. }
$$

Thus the quantum dimension of $V$ is given by

$$
\operatorname{trace}_{V}\left(h^{2}\right)=q^{2 m}+q^{2(m-2)}+\cdots+q^{-2 m} .
$$

\section{Tannaka-Krein Duality}

The Tannaka-Krein type duality has been already established by Woronowicz based on his formulation of compact quantum groups. In this section, we present a dualized version of the corresponding theorem for compact quantum groups. The assumption of the existence of the characteristic element may restrict the class of compact quantum groups but we can give a simpler proof of the Tannaka-Krein duality.

Definition 4.1. $A{ }^{*}$-monoidal category $\mathscr{R}$ with $\varepsilon$-structure $\left\{\varepsilon_{V}\right\}$ is called unitary representation theory if

(i) objects of $\mathscr{R}$ are finite dimensional Hilbert spaces,

(ii) $\operatorname{Hom}(V, M)$ in $\mathscr{R}$ is a linear subspace of the vector space $\mathscr{L}(V, W)$ of linear maps from $V$ into $W$ with ${ }^{*}$-operation inherited from $\mathscr{L}(V, W)$,

(iii) the monoidal structure is also inherited from $\mathscr{L}(V, W)$ : for $V, V^{\prime}, W, W^{\prime} \in O b$ ject and $S \in \operatorname{Hom}\left(V, V^{\prime}\right), T \in H o m\left(W, W^{\prime}\right)$, the monoidal operation $V \otimes W$ is given by ordinary tensor product of Hilbert spaces and $S \otimes T \in H o m(V \otimes W$, $\left.V^{\prime} \otimes W^{\prime}\right)$ is given by the ordinary tensor product of linear maps. The unit object is given by $\mathbb{C}$,

(iv) $V^{*}$ is the dual space of $V$ with the conjugation of $T \in H o m(V, W)$ given by

$$
\bar{T} v^{*}=(T v)^{*}, \quad v \in V,
$$

(recall that $v^{*} \in V^{*}$ is the linear form on $V$ obtained from $v \in V$ by the inner product in $V$ ), 
(v) the $\varepsilon$-structure $\left\{\varepsilon_{V}\right\}$ is positive in the sense that

$$
\varepsilon_{V}\left(v \otimes v^{*}\right) \geqq 0 \quad \text { for } \forall V \text { and } \forall v \in V .
$$

With this terminology, the result in Sect. 3 is summarized in the following form: the monoidal category of finite dimensional unitary representations of a compact quantum group gives a unitary representation theory.

Now conversely suppose that we are given a unitary representation theory $\mathscr{R}$. Since $\operatorname{End}(V)$ is a ${ }^{*}$-subalgebra of $\mathscr{B}(V)$, adding subspaces corresponding to projections in $\operatorname{End}(V)$ to objects if necessary, we may assume that every object in $\mathscr{R}$ is a direct sum of irreducible ones. Denote by $\mathscr{R}$ the set of equivalence classes of irreducible objects in $\mathscr{R}$ and set

$$
\tilde{\mathscr{M}} \equiv\left\{x=\left\{x_{V}\right\}_{V \in \text { Object }} ; x_{V} \in \mathscr{B}(V) \text { and } T x_{V}=x_{W} T \text { if } T \in \operatorname{Hom}(V, W)\right\} \text {, }
$$

which is a $*$-algebra by object-wise operations. Take a set of representatives of $\widehat{\mathscr{R}}$ and simply write $V \in \hat{\mathscr{R}}$ if $V$ is in that set. Then it is easy to check that

$$
\tilde{\mathscr{M}} \ni x \mapsto\left\{x_{V}\right\}_{V \in \hat{\mathscr{M}}} \in \prod_{V \in \hat{\mathscr{M}}} \mathscr{B}(V)
$$

is a bijection and, if we set

$$
\begin{aligned}
& \mathscr{M}=\left\{x \in \tilde{\mathscr{M}} ; x_{V}=0 \text { for } V \in \widehat{\mathscr{R}} \text { except for finitely many } V \text { 's in } \widehat{\mathscr{R}}\right\}, \\
& M=\left\{x \in \tilde{\mathscr{M}} ; \sup _{V \in \hat{\mathscr{R}}}\left\|x_{V}\right\|<+\infty\right\},
\end{aligned}
$$

then $\mathscr{M}$ and $M$ do not depend on the choice of representatives of $\hat{\mathscr{R}}$.

Clearly $M$ is a (discrete) von Neumann algebra with $\mathscr{M}$ a dense *-subalgebra, which is identified with $\bigoplus_{V \in \hat{\mathscr{B}} \mathscr{B}} \mathscr{B}(V)$ through the above isomorphism on occasion.

Define a comultiplication $\Delta$ in $M$ by

$$
\Delta(x)=\bigoplus_{V_{1}, V_{2} \in \hat{\mathscr{R}}}\left(\sum_{T: V \rightarrow V_{1} \otimes V_{2}} T x T^{*}\right),
$$

which is sitting in

$$
\begin{aligned}
& \bigoplus_{V_{1}, V_{2} \in \hat{\mathscr{M}}} \mathscr{B}\left(V_{1} \otimes V_{2}\right)=\left(\bigoplus_{V_{1} \in \hat{\mathscr{M}}} \mathscr{B}\left(V_{1}\right)\right) \otimes\left(\bigoplus_{V_{2} \in \hat{\mathscr{B}}} \mathscr{B}\left(V_{2}\right)\right) \\
& \cong M \otimes M
\end{aligned}
$$

Here $V$ and $T: V \rightarrow V_{1} \otimes V_{2}$ runs through a family of isometric intertwiners which gives an irreducible decomposition of $V_{1} \otimes V_{2}$. It is immediate to see that the above formula gives a ${ }^{*}$-isomorphism from $M$ into $M \otimes M$ with $\Delta\left(1_{M}\right)=1_{M} \otimes 1_{M}$. It is also easy to check that $\Delta$ depends on neither choices of representatives in $\widehat{\mathscr{R}}$ nor choices of intertwiners in the decomposition of $V_{1} \otimes V_{2}$.

More generally we can show the following:

Lemma 4.2. Define a linear map $\Delta^{(n-1)}: M \rightarrow M \otimes \cdots \otimes M b y$

$$
\begin{aligned}
\Delta^{(n-1)}(x) & =\bigoplus_{V_{1}, \ldots, V_{n} \in \hat{\mathscr{B}}}\left(\sum_{T: V \rightarrow V_{1} \otimes \ldots \otimes V_{n}} T x T^{*}\right) \\
& \in \bigoplus_{V_{1}, \ldots, V_{n} \in \hat{\mathscr{B}}} \mathscr{B}\left(V_{1} \otimes \cdots \otimes V_{n}\right) \cong M \otimes \cdots \otimes M .
\end{aligned}
$$


Then $\Delta^{(n-1)}$ is $a^{*}$-isomorphism and independent of the choice of intertwiners in the decomposition of $V_{1} \otimes \cdots \otimes V_{n}$.

Remark. If $x \in M$ is supported by $V \in \widehat{\mathscr{R}}$, then the component of $\Delta^{(n-1)}(x)$ in

$$
\mathscr{B}\left(V_{1} \otimes \cdots \otimes V_{n}\right)=\mathscr{B}\left(V_{1}\right) \otimes \cdots \otimes \mathscr{B}\left(V_{n}\right) \subset M \otimes \cdots \otimes M
$$

is given by

$$
\Delta^{(n-1)}(x)= \begin{cases}\sum_{T} T x T^{*} & \text { if } V \prec V_{1} \otimes \cdots \otimes V_{n} \\ 0 & \text { otherwise } .\end{cases}
$$

Corollary 4.3. $\triangle$ gives a comultiplication.

$\because)$ Write

$$
\Delta(x)=\bigoplus_{V_{1}, V_{23} \in \hat{\mathscr{R}}}\left(\sum_{T: V \rightarrow V_{1} \otimes V_{23}} T x T^{*}\right) .
$$

Then

$$
\begin{aligned}
(1 \otimes \Delta) \Delta(x) & =\bigoplus_{V_{1}, V_{2}, V_{3} \in \hat{\mathscr{A}}} \sum_{S: V_{23} \rightarrow V_{2} \otimes V_{3}}(1 \otimes S)\left(\sum_{T: V \rightarrow V_{1} \otimes V_{23}} T x T^{*}\right)(1 \otimes S)^{*} \\
& =\bigoplus_{V_{1}, V_{2}, V_{3} \in \hat{\mathscr{R}}} \sum_{S, T}(1 \otimes S) T x T^{*}\left(1 \otimes S^{*}\right) \\
& =\Delta^{(2)}(x) .
\end{aligned}
$$

In the last line, we have used the fact that $\{(1 \otimes S) T\}_{S, T}$ gives an irreducible decomposition of $V_{1} \otimes V_{2} \otimes V_{3}$.

Similarly we have $(\Delta \otimes 1) \Delta(x)=\Delta^{(2)}(x)$.

Remark. If we understand the meaning of the tensor product $\overline{\mathscr{M}} \otimes \widetilde{\mathscr{M}}$ as the vector space which is isomorphic to the set of doubly indexed sequences of finite-dimensional operators in $\{\mathscr{B}(V) \otimes \mathscr{B}(W)\}_{V, W \in \hat{\mathscr{K}}}$, then the above $\Delta$ is obviously extended to the injective ${ }^{*}$-homomorphism $\tilde{\mathscr{M}} \rightarrow \tilde{\mathscr{M}} \otimes \tilde{\mathscr{M}}$ and gives a commultiplication in $\tilde{\mathscr{M}}$.

The counit of $\Delta$ is given by the evaluation map

$$
\varepsilon: M \ni x \mapsto x_{1} \in \mathbb{C} .
$$

Here 1 denotes the unit object which is nothing but $\mathbb{C}$ as a vector space. Since $\varepsilon$ is obviously a normal *-homomorphism, we have obtained a $\mathrm{W}^{*}$-bialgebra $(M, \Delta, \varepsilon)$.

Next we define a transposition $\tau$ in $M$ as the ordinary transposition in $\oplus V \in \hat{\mathscr{R}} \mathscr{B}(V)$ :

$$
\tau(x)_{V}={ }^{t}\left(x_{V^{*}}\right), \quad x \in \mathscr{M} .
$$

Clearly $\tau$ is an involutive antimultiplicative map. Its anticomultiplicativity is checked by

$$
\begin{aligned}
\sim \circ(\tau \otimes \tau) \circ \Delta(x) & =\bigoplus_{V_{1}, V_{2}} \sum_{T: V \rightarrow V_{1} \otimes V_{2}}{ }^{t}\left(T x T^{*}\right) \\
& =\underset{V_{1}, V_{2}}{\bigoplus} \sum_{T} \bar{T}^{t} x \bar{T}^{*} \\
& =\Delta(\tau(x)) .
\end{aligned}
$$

Note that $\left\{\bar{T}: V^{*} \rightarrow V_{2}^{*} \otimes V_{1}^{*}\right\}$ gives a decomposition of $V_{2}^{*} \otimes V_{1}^{*}$. 
Let us now construct a characteristic element of the Hopf-von Neumann algebra $(M, \Delta, \varepsilon, \tau)$. First, to each $\varepsilon_{V}^{*}(1) \in V \otimes V^{*}$, associate a positive invertible operator $h_{V}$ in $\mathscr{B}(V)$ by

$$
\left(\varepsilon_{V}^{*}(1) \mid v \otimes w^{*}\right)=\left(w \mid h_{V}(v)\right), \quad v, w \in V
$$

(the qualified properties follow from the faithfullness and the positivity of the $\varepsilon$-structure $\left\{\varepsilon_{V}\right\}$ ).

Lemma 4.4. Let $h_{V}^{i t}, t \in \mathbb{R}$ be the exponentiated unitary operator of $h_{V}$. Then the family $h^{i t}=\left\{h_{V}^{i t}\right\}_{V}$ belongs to $M$ and satisfies $(i) \Delta\left(h^{i t}\right)=h^{i t} \otimes h^{i t}$ and (ii) $\tau\left(h^{i t}\right)=h^{-i t}$.

$\because)$ From the property of $\varepsilon$-structure

$$
(T \otimes 1) \varepsilon_{V}^{*}=\left(1 \otimes{ }^{t} T\right) \varepsilon_{W}^{*} \quad \text { for } T: V \rightarrow W,
$$

we obtain the relation on operators $h_{V}$;

$$
T h_{V}=h_{W} T
$$

which implies

$$
T h_{V}^{i t}=h_{W}^{i t} T \text {. }
$$

Thus the family $\left\{h_{V}^{i t}\right\}$ defines an element $h^{i t}$ in $M$.

The multiplicativity of $\varepsilon$-structure $\varepsilon_{V_{1} \otimes V_{2}}=\varepsilon_{V_{1}}\left(1_{V_{1}} \otimes \varepsilon_{V_{2}} \otimes 1_{V_{1}^{*}}\right)$ entails the operator relation $h_{V_{1} \otimes V_{2}}=h_{V_{1}} \otimes h_{V_{2}}$ which in turn implies

$$
h_{V_{1} \otimes V_{2}}^{i t}=h_{V_{1}}^{i t} \otimes h_{V_{2}}^{i t} \text {. }
$$

Using these relations,

$$
\begin{aligned}
\Delta\left(h^{i t}\right) & =\bigoplus_{V_{1}, V_{2}} \sum_{T: V \rightarrow V_{1} \otimes V_{2}} T h_{V}^{i t} T^{*} \\
& =\bigoplus_{V_{1}, V_{2}} h_{V_{1} \otimes V_{2}}^{i t} \quad \text { (use the additivity of } \varepsilon \text {-structure) } \\
& =\underset{V_{1}, V_{2}}{\bigoplus} h_{V_{1}}^{i t} \otimes h_{V_{2}}^{i t} \\
& =h^{i t} \otimes h^{i t}
\end{aligned}
$$

which proves (i).

The desired relation in (ii) is equivalent to

$$
\left(h_{V^{*}}^{i t} v^{*}\right)^{*}=h_{V}^{i t} v \quad \text { for } v \in V,
$$

which is furthermore equivalent to

$$
\left(h_{V} v\right)^{*}=h_{V^{*}}^{-1} v^{*}, \quad v \in V
$$

by analytic continuation.

Now this last relation follows from the identity

$$
\left(\varepsilon_{V}^{*} \otimes 1\right)^{*}\left(1 \otimes \varepsilon_{V^{*}}^{*}\right)=1_{V}
$$


mentioned in the last part of Notation and Terminology. In fact, making use of this identity, we can deduce as follows:

$$
\begin{aligned}
\left(w^{*} \mid v^{*}\right)=(v \mid w) & =\left(\left(\varepsilon_{V}^{*} \otimes 1\right) v \mid\left(1 \otimes \varepsilon_{V^{*}}^{*}\right) w\right) \\
& =\left(\varepsilon_{V}^{*}(1) \otimes v \mid w \otimes \varepsilon_{V^{*}}^{*}(1)\right) \\
& =\left(\varepsilon_{V}^{*}(1) \mid w \otimes h_{V^{*}} v^{*}\right) \\
& =\left(\left(h_{V^{*}} v^{*}\right)^{*} \mid h_{V} w\right) \\
& =\left(\left(h_{V} w\right)^{*} \mid h_{V^{*}} v^{*}\right)
\end{aligned}
$$

which means

$$
h_{V^{*}}\left(h_{V} w\right)^{*}=w^{*}, \quad w \in V .
$$

Remark. At the starting definition of unitary representation theory, we may allow possibly infinite dimensional representations. Even in that case, the assumed $\varepsilon$-structure gives rise to a family of positive Hilbert-Schmidt operators $\left\{h_{V}\right\}$. The last part in the above proof then shows that $h_{V}$ must have the bounded inverse, whence the allowed representation spaces must be finite-dimensional.

Finally we check that the defining relation of antipode is satisfied by

$$
\tau \circ(A d h)=(A d h) \circ \tau \text {. }
$$

Here $h=\left\{h_{V}\right\}$ is a positive invertible element in $\tilde{\mathscr{M}}$ and the adjoint $A d h$ is taken on $\tilde{\mathscr{M}}$. Although this operator does not preserve $M$ generally, the dense *-subalgebra $\mathscr{M}$ is invariant under $A d h$ and can be used as the defining domain of $A d h$. Note that, thanks to the algebraic nature of the von Neumann algebra $M$, there is no analytical difficulty in dealing with the unbounded operator algebra $\tilde{\mathscr{M}}$.

Since $\tau(x)_{W}={ }^{t}\left(x_{W^{*}}\right)$ for $x \in M$ and $h=\left\{h_{W}\right\}$, the $W$-component of $m(1 \otimes \tau \circ A d h) \Delta(x)$ is given by

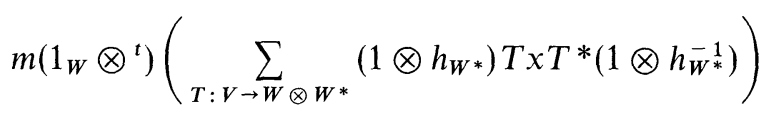

for $W \in \widetilde{R}$. Here $m$ denotes the (object-wise) multiplication map $M \otimes M \rightarrow M$. have

Take an orthonormal basis $\left\{w_{j}\right\}$ in $W$. Then for $y \in \mathscr{B}(W)$ and $z \in \mathscr{B}\left(W^{*}\right)$, we

$$
\begin{aligned}
\left(w^{\prime} \mid m\left(y \otimes{ }^{t} z\right) w\right) & =\sum_{j}\left(w^{\prime} \otimes w_{j} \mid\left(y \otimes{ }^{t} z\right)\left(w_{j} \otimes w\right)\right) \\
& =\sum_{j}\left(w^{\prime} \mid y w_{j}\right)\left(\left.w_{j}\right|^{t} z w\right) \\
& =\sum_{j}\left(w^{\prime} \mid y w_{j}\right)\left(w^{*} \mid z w_{j}^{*}\right) \\
& =\sum_{j}\left(w^{\prime} \otimes w^{*} \mid(y \otimes z)\left(w_{j} \otimes w_{j}^{*}\right)\right) .
\end{aligned}
$$


Using this relation, we now calculate as

$$
\begin{aligned}
\left(w^{\prime} \mid\right. & {[m(1 \otimes \tau \circ \operatorname{Adh}) \Delta(x)] w) } \\
& =\sum_{j}\left(w^{\prime} \otimes w^{*} \mid \sum_{T: V \rightarrow W} \otimes W^{*}\right. \\
\quad= & \sum_{T: V \rightarrow W \otimes W^{*}}\left(w^{\prime} \otimes w^{*} \mid\left(1 \otimes h_{W^{*}}\right) T x T^{*}\left(1 \otimes h_{W^{*}}\right) T x_{V} T^{*} \varepsilon_{W}^{*}(1)\right) \\
& =\left(w^{\prime} \otimes w_{j} \otimes \mid\left(1 \otimes w_{j}^{*}\right)\right)
\end{aligned}
$$

(here $T: \mathbf{1} \rightarrow W \otimes W^{*}$ and note that $T^{*} \varepsilon_{W}^{*}(1)=0$ if $V \not \equiv \mathbf{1}$ )

$$
\begin{aligned}
& =\varepsilon(x)\left(w^{\prime} \otimes w^{*} \mid\left(1 \otimes h_{W^{*}}\right) T T^{*} \varepsilon_{W}^{*}(1)\right) \\
& =\varepsilon(x)\left(w^{\prime} \otimes w^{*} \mid\left(1 \otimes h_{W^{*}}\right) \varepsilon_{W}^{*}(1)\right)
\end{aligned}
$$

$\left(T T^{*}\right.$ is the range projection to $\left.\varepsilon_{W}^{*}(1)\right)$

$$
\begin{aligned}
& =\varepsilon(x) \sum_{j}\left(w^{\prime} \otimes w^{*} \mid\left(1 \otimes h_{W *}\right)\left(1 \otimes h_{W *}^{-1}\right)\left(w_{j} \otimes w_{j}^{*}\right)\right) \\
& =\varepsilon(x)\left(w^{\prime} \mid w\right)
\end{aligned}
$$

proving that the $W$-component of $m(1 \otimes \tau \circ A d h) \Delta(x)$ is given by $\varepsilon(x) 1_{W}$, i.e.,

$$
m(1 \otimes \tau \circ A d h) \Delta(x)=\varepsilon(x) 1_{M} .
$$

Similarly we can show that $m(\tau \circ \operatorname{Adh} \otimes 1) \Delta(x)=\varepsilon(x) 1_{M}$.

In this way we have obtained the compact quantum group von Neumann algebra $\left(M, \Delta, \varepsilon, \tau,\left\{h^{i t}\right\}\right)$. By the construction, the starting unitary representation theory coincides with (a full subcategory of) the unitary representation theory of $\left(M, \Delta, \varepsilon, \tau,\left\{h^{i t}\right\}\right)$.

Theorem 4.5. Any unitary representation theory is given by finite dimensional unitary representations of a uniquely determined compact quantum group-von Neumann algebra.

Remark. If we start with a Hopf *-algebra $A$ with characteristic element, the Hopf-von Neumann algebra constructed from the unitary representation theory of $A$ can be interpreted as the $\mathrm{W}^{*}$-completion of $A$.

\section{Invariant Weights}

Let $(M, \Delta, \varepsilon)$ be a compact quantum group-von Neumann algebra. A weight $\omega$ of $M$ is called the left Haar weight if

$$
\langle(1 \otimes x) \Delta(y)\rangle_{1 \otimes \omega}=\sigma\left(\langle\Delta(x)(1 \otimes y)\rangle_{1 \otimes \omega}\right), \quad x, y \in \mathscr{M} .
$$

Here $\langle\cdot\rangle_{1 \otimes \omega}$ denotes the slice map $\mathscr{M} \otimes \mathscr{M}$ relative to the restriction of $\omega$ to $\mathscr{M}$ and $\sigma=\tau \circ \theta_{-1}$ denotes an antipode-like operation in $\mathscr{M}$ (cf. Definition 1.2 and the following remarks). Note that $(1 \otimes x) \Delta(y)$ and $\Delta(x)(1 \otimes y)$ belong to $\mathscr{M} \otimes \mathscr{M}$ 
for $x, y \in \mathscr{M}$. Similarly a right Haar weight is defined to be a weight $\omega$ fulfilling

$$
\langle(x \otimes 1) \Delta(y)\rangle_{\omega \otimes 1}=\sigma^{-1}\left(\langle\Delta(x)(y \otimes 1)\rangle_{\omega \otimes 1}\right), \quad x, y \in \mathscr{M} .
$$

The existence and uniqueness for Haar weights are established by Effros and Ruan for "discrete quantum groups" ([ER]) which are essentially the same class as our compact groups arising from unitary representation theories. These are also checked by Masuda and Nakagami for the group von Neumann algebra of $S U_{q}(n)$ $([\mathrm{MN}])$, where a more general definition for Haar weights is also provided.

Here we present a proof of the existence and uniqueness of invariant weights for the quantum group-von Neumann algebra $M$ arising from a unitary representation theory as an applicability of the present formalism. Remark here that we have to take care of the domain problem in an invariant wieght on the von Neumann algebra as well as the meaning of its invariance generically. In the present situation, however, due to the specific structure of $M$, we can avoid the analytical complexity by replacing $M$ with the dense *-algebra $\mathscr{M}$ of $M$. Then the above definition for the invariance of weights makes sense by taking $x, y \in \mathscr{M}$.

Recall that $M$ is isomorphic to $\bigoplus_{V \in \mathscr{\mathscr { R }} \mathscr{B}}(V)$ as a von Neumann algebra and $\mathscr{M}$ is identified with the algebraic sum. Hence any weight $w$ on $M$ is determined by the restriction to $\mathscr{M}$ and takes the form

$$
w(x)=\sum_{V \in \hat{\mathscr{M}}}\left\langle\rho_{V} x_{V}\right\rangle
$$

with $\rho_{V}$ a positive operator in $\mathscr{B}(V)$ (the so-called density matrix). Here $\langle\cdot\rangle$ denotes the ordinary trace in matrix algebras.

First we show the uniqueness: Take $x$ and $y \in M$ in the definition of right invariance so that $x$ and $y$ are supported by $\mathbb{C}_{\varepsilon}$ and $W \in \widehat{\mathscr{R}}$ respectively, and then compare the $W$-component:

$$
\begin{gathered}
\begin{aligned}
\text { 1.h.s. } & =\left[\langle(x \otimes 1) \Delta(y)\rangle_{\omega \otimes 1}\right]_{W} \\
= & \left\langle\left(x_{1} \otimes 1\right) \Delta(y)_{1 \otimes W}\right\rangle_{\omega \otimes 1} \\
= & \left\langle x_{1} \otimes y_{W}\right\rangle_{\omega \otimes 1} \\
& =\rho_{1} x_{1} y_{W}, \\
\text { r.h.s. }= & {\left[h^{-1} \tau\left(\langle\Delta(x)(y \otimes 1)\rangle_{\omega \otimes 1}\right) h\right]_{W} } \\
= & h_{W}^{-1 t}\left(\left[\langle\Delta(x)(y \otimes 1)\rangle_{\omega \otimes 1}\right]_{W^{*}}\right) h_{W} \\
= & h_{W}^{-1 t}\left(\left\langle T x_{1} T^{*}\left(y_{W} \rho_{W} \otimes 1\right)\right\rangle_{W \otimes 1}\right) h_{W} .
\end{aligned}
\end{gathered}
$$

Here $\langle\cdot\rangle_{W \otimes 1}$ refers to the partial trace in the $W$-component and $T \in$ $\operatorname{Hom}\left(\mathbb{C}_{\varepsilon}, W \otimes W^{*}\right)$ is an isometry. To rewrite the inside of the transposition, we calculate for $u, w \in W$ as follows:

$$
\begin{aligned}
\left(\left.u\right|^{t}\left(\left\langle T x_{1} T^{*}\left(y_{W} \rho_{W} \otimes 1\right)\right\rangle_{W \otimes 1}\right) w\right) & =\left(w^{*} \mid\left(\left\langle T x_{1} T^{*}\left(y_{W} \rho_{W} \otimes 1\right)_{W \otimes 1}\right) u^{*}\right)\right. \\
& =x_{1} \sum_{j}\left(w_{j} \otimes w^{*} \mid T T^{*}\left(y_{W} \rho_{W} \otimes 1\right)\left(w_{j} \otimes u^{*}\right)\right)
\end{aligned}
$$




$$
\begin{aligned}
& =x_{1} d(W)^{-1} \sum_{j}\left(w_{j} \otimes w^{*} \mid \varepsilon_{W}^{*} \varepsilon_{W}\left(y_{W} \rho_{W} w_{j} \otimes u^{*}\right)\right) \\
& =x_{1} d(W)^{-1} \sum_{j}\left(h_{W} w_{j} \mid w\right)\left(u \mid h_{W} y_{W} \rho_{W} w_{j}\right) \\
& =x_{1} d(W)^{-1}\left(u \mid h_{W} y_{W} \rho_{W} h_{W} w\right),
\end{aligned}
$$

which shows that

$$
\text { r.h.s. }=x_{1} d(W)^{-1} y_{W} \rho_{W} h_{W}^{2} .
$$

Now comparing the left and right sides, we conclude that

$$
\rho_{W}=\rho_{1} d(W) h_{W}^{-2},
$$

proving the uniqueness of right invariant weights.

Now conversely suppose that the weight $\omega$ on $M$ is defined by

$$
\omega(x)=\sum_{V \in \hat{\mathscr{R}}} d(V)\left\langle h_{V}^{-2} x_{V}\right\rangle .
$$

To show the right invariance, we may assume that $x$ and $y \in \mathscr{M}$ are supported by $V$ and $W \in \widehat{\mathscr{R}}$ respectively. Then for $U \in \widehat{\mathscr{R}}$,

$$
\left[\langle(x \otimes 1) \Delta(y)\rangle_{\omega \otimes 1}\right]_{U}=d(V) \sum_{T: W \rightarrow V \otimes U}\left\langle\left(h_{V}^{-2} x_{V} \otimes 1\right) T y_{W} T^{*}\right\rangle_{V \otimes 1} .
$$

Let $R: V \rightarrow W \otimes U^{*}$ be the right Frobenius transform of $T^{*}$, i.e., $R=$ $\left(T^{*} \otimes 1_{U^{*}}\right)\left(1_{V} \otimes \varepsilon_{U}^{*}\right)$. Then $T^{*}=\left(1 \otimes \varepsilon_{U^{*}}\right)(R \otimes 1)$ and we have

$\left.[\langle(x \otimes 1) \Delta y)\rangle_{\omega \otimes 1}\right]_{U}$

$$
=d(V) \sum_{R: V \rightarrow W \otimes U^{*}}\left\langle\left(x_{V} \otimes 1\right)\left(R^{*} \otimes 1\right)\left(1 \otimes \varepsilon_{U^{*}}^{*}\right) y_{W}\left(1 \otimes \varepsilon_{U^{*}}\right)(R \otimes 1)\left(h_{V}^{-2} \otimes 1\right)\right\rangle_{V \otimes 1}
$$

(note that $R h_{V}^{-2} R^{*}=h_{W \otimes U^{*}}^{-2}$ )

$$
\begin{aligned}
= & d(V) \sum_{R}\left\langle\left(x_{V} R^{*} \otimes 1\right)\left(y_{W} \otimes 1 \otimes 1\right)\left(1 \otimes \varepsilon_{U^{*}}^{*} \varepsilon_{U^{*}}\right)\left(h_{W}^{-2} \otimes U^{*} \otimes 1\right)(R \otimes 1)\right\rangle_{V \otimes 1} \\
= & d(V) \sum_{R}\left\langle\left(R x_{V} R^{*} \otimes 1\right)\left(y_{W} \otimes 1 \otimes 1\right)\left(1 \otimes \varepsilon_{U}^{*} \varepsilon_{U^{*}}\right)\right. \\
& \left.\times\left(1 \otimes h_{U^{*}}^{-2} \otimes 1\right)\left(h_{W}^{-2} \otimes 1 \otimes 1\right)\right\rangle_{W} \otimes U^{*} \otimes 1
\end{aligned}
$$

(note that $h_{W \otimes U^{*}}^{-2}=h_{W}^{-2} \otimes h_{U^{*}}^{-2}$ ).

Since $\left\{R_{0}=d(V)^{1 / 2} d(W)^{-1 / 2} R\right\}$ are isometries and give a decomposition of $W \otimes U^{*}$ into pair-wise orthogonal $V$-components (see [FRTC, Lemma 4.8] and [NOA, Corollary 1.6]), the last equation is further computed as

$$
\begin{aligned}
= & d(W) \sum_{R_{0}: V \rightarrow W \otimes U^{*}}\left\langle\left(\left[\left(h_{W}^{-2} \otimes 1\right) R_{0} x_{V} R_{0}^{*}\left(y_{W} \otimes 1\right)\right] \otimes 1\right)\right. \\
& \left.\times\left(1 \otimes \varepsilon_{U^{*}}^{*} \varepsilon_{U^{*}}\right)\left(1 \otimes h_{U^{*}}^{-2} \otimes 1\right)\right\rangle_{W \otimes U^{*} \otimes 1} \\
& \left(\text { putting } z=\langle\Delta(x)(y \otimes 1)\rangle_{\omega \otimes 1} \in M\right) \\
= & \left\langle\left(z_{U^{*}} \otimes 1\right) \varepsilon_{U^{*}}^{*} \varepsilon_{U^{*}}\left(h_{U^{*}}^{-2} \otimes 1\right)\right\rangle_{U^{*} \otimes 1} .
\end{aligned}
$$


Now take $u, u^{\prime} \in U$ and calculate as

$$
\begin{aligned}
& \left(u \mid\left\langle\left(z_{U^{*}} \otimes 1\right) \varepsilon_{U^{*} \varepsilon_{U^{*}}}\left(h_{U^{*}}^{-2} \otimes 1\right)\right\rangle_{U^{*} \otimes 1} u^{\prime}\right) \\
& =\sum_{j}\left(u_{j}^{*} \otimes u \mid\left(z_{U^{*}} \otimes 1\right) \varepsilon_{U^{*}}^{*} \varepsilon_{U^{*}}\left(h_{U^{*}}^{-2} \otimes 1\right)\left(u_{j}^{*} \otimes u^{\prime}\right)\right) \\
& =\sum_{j}\left(z_{U^{*}}^{*} u_{j}^{*} \otimes u \mid \varepsilon_{U^{*}}^{*}(1)\right)\left(\varepsilon_{U^{*}}^{*}(1) \mid h_{U^{*}}^{-2} u_{j}^{*} \otimes u^{\prime}\right) \\
& =\sum_{j}\left(u_{j}^{*} \mid z_{U^{*}} h_{U^{*}} u^{*}\right)\left(h_{U^{*}}^{-1} u^{\prime *} \mid u_{j}^{*}\right) \\
& =\left(u^{\prime *} \mid h_{U^{*}}^{-1} z_{U^{*}} h_{U^{*}} u^{*}\right) \\
& =\left(u^{*} \mid \overline{\left(h_{U^{*}}^{-1} z_{U^{*}} h_{U^{*}} u\right)^{*}}\right) \\
& =\left(h_{U} \overline{z_{U^{*}}} h_{U}^{-1} u \mid u^{\prime}\right) \\
& =\left(u \mid h_{U}^{-1} \tau(z)_{U} h_{U} u^{\prime}\right) \text {. }
\end{aligned}
$$

Thus we have obtained

$$
\begin{aligned}
{\left[\langle(x \otimes 1) \Delta(y)\rangle_{\omega \otimes 1}\right]_{U} } & =h_{U}^{-1} \tau\left(\langle\Delta(x)(y \otimes 1)\rangle_{\omega \otimes 1}\right) h_{U} \\
& =\sigma^{-1}\left(\langle\Delta(x)(y \otimes 1)\rangle_{\omega \otimes 1}\right)
\end{aligned}
$$

for $x, y \in \mathscr{M}$, proving the right invariance of $\omega$.

Similar calculations work for the left invariance of weights and we finally obtain the following result:

Theorem 5.1. Let $\left(M, \Delta, \varepsilon, \tau,\left\{h^{i t}\right\}\right)$ be the quantum group-von Neumann algebra associated to a unitary representation theory $\mathscr{R}$. Then there exists right (resp. left) Haar weights $\omega_{R}$ (resp. $\omega_{L}$ ) on $M$, which are unique up to a scalar multiple and given by the following formula:

and

$$
\omega_{R}(x)=\sum_{V \in \hat{\mathscr{R}}} d(V)\left\langle h_{V}^{-2} x_{V}\right\rangle
$$

$$
\omega_{L}(x)=\underset{V \in \hat{\mathscr{R}}}{ } d\left(V^{*}\right)\left\langle h_{V}^{2} x_{V}\right\rangle .
$$

Here $d(V)$ denotes the quantum dimension of $V,\langle\cdot\rangle$ denotes the ordinary trace, and $h_{V}^{ \pm 2}$ is the square or the squared inverse of the positive invertible operators in $\mathscr{B}(V)$ associated to the $\varepsilon$-structure

Remark. (i) The above formula for the invariant weights has been known in the context of compact matrix quantum groups and their Pontryagin duals ([PW]).

(ii) If the unitary representation theory is the one given by finite dimensional unitary representation of a compact group $G$, then the above for the invariant weight is reduced to the one for Plancherel weight (note that $d(V)=\operatorname{dim} V$ and $h_{V}=1_{V}$ in this case). In this respect, it would be appropriate to call them Plancherel weight rather than Haar weight.

(iii) A weight $\omega$ on a compact quantum group-von Neumann algebra is called left (resp. right) invariant if

$$
\left.\langle\Delta(x)\rangle_{1 \otimes \omega}=\omega(x) 1 \quad \text { (resp. }\langle\Delta(x)\rangle_{\omega \otimes 1}=\omega(x) 1\right), \text { for } x \in \mathscr{M} .
$$


Owing to the results in [VD], this condition is sufficient to deduce the strong invariance required in the definition of Haar weights.

(iv) With the above explicit formula, it is easy to check the requirements of Haar weights in [MN, Definition 1.1] for our somewhat simple-minded Haar weights.

Corollary 5.2. For a right-invariant weight $\omega$ on $M$, the weight $\omega \circ \tau$ is left-invariant. If $d\left(V^{*}\right)=d(V)$ for any irreducible $V$, we further have

$$
\omega(\tau(x))=\omega\left(x h^{4}\right), \quad x \in M_{+} .
$$

$\because$ ) The first assertion is a consequence of the fact that the conditions of left and right invariances on weights are interchanged by applying the transposition $\tau$.

To see the second assertion, first recall the definitions $\tau(x)_{V}={ }^{t}\left(x_{V^{*}}\right),\left(h^{i t}\right)_{V}=h_{V}^{i t}$ and then calculate as follows:

$$
\begin{aligned}
\omega(\tau(x)) & =\sum_{V} d(V)\left\langle h_{V}^{-2 t} x_{V^{*}}\right\rangle=\sum_{V} d(V) \sum_{j}\left(\overline{x_{V^{*}}} v_{j} \mid h_{V}^{-2} v_{j}\right) \\
& =\sum_{V} d(V) \sum_{j}\left(\left(h_{V}^{-2} v_{j}\right)^{*} \mid x_{V^{*}} v_{j}^{*}\right)=\sum_{V} d(V) \sum_{j}\left(h_{V^{*}}^{2} v_{j}^{*} \mid x_{V^{*}} v_{j}^{*}\right) \\
& =\sum_{V} d\left(V^{*}\right)\left\langle h_{V^{*}}^{2} x_{V^{*}}\right\rangle=\sum_{V} d(V)\left\langle h_{V}^{2} x_{V}\right\rangle \\
& =\omega\left(h^{4} x\right)=\omega\left(x h^{4}\right) .
\end{aligned}
$$

\section{References}

[BS] Baaj, S., Skandalis, G.: Unitaires multiplicatifs et dualite pour les produits croises de $C^{*}$-algebre. Annal. Sci. Ec. Norm. Sup 26, 425-488 (1993)

[Dr] Doplicher, S., Roberts, J.E.: A new duality theory for compact groups. Invent. Math. 98, 157-218 (1989)

[DR] Drinfel'd, V.G.: Quantum groups. In Proceedings of ICM (1987), Berkeley

[DJN] Durhuus, B., Jakobsen, H.P., Nest, R.: Topological quantum field theories from generalized 6j-symbol. Rev. Math. Phys. 5, 1-67 (1993)

[ER] Effros, E.G., Ruan, Z.-J.: Discrete quantum groups, I. The Haar measure Preprint (1993)

[ES] Enock, M., Schwartz, J.M.: Kac Algebra and Duality of Locally Compact Group Berlin Heidelberg New York: Springer, 1992

[EK] Evans, D., Kawahigashi, Y.: Orbifold subfactors from Hecke algebras. Preprint (1992)

[Ji] Jimbo, M.: A $q$-difference analogue of $U(\mathfrak{g})$ and the Yang-Baxter equation. Lett. Math. Phys. 10, 63-69 (1985)

[Jo] Jones, V.F.R.: Subfactors and Knots. Regional Conference Series in Math. No. 80, Providence RI: American Mathematical Society, 1991

[L] Lusztig, G.: Quantum deformations of certain simple modules over enveloping algebras. Adv. Math. 70, 237-249 (1988)

[M] MacLane, S.: Natural associativity and commutativity. Rice Univ. Studies 49, 28-46 (1963)

[MN] Masuda, T., Nakagami, Y.: A von Neumann algebra framework for the duality of the quantum groups. Preprint (1993)

[N] Nakagami, Y.: Takesaki duality for the crossed product by quantum groups. In: Perspectives on Quantum and Non-Commutative Analysis, (H. Araki and A. Kishimoto, eds.), Basel-bottom Birkhauser, 1993

[PW] Podles̀, P., Woronowicz, S.L.: Quantum deformation of Lorentz group. Commun. Math. Phys. 130, 381-431 (1990) 
[R1] Rosso, M.: Finite-dimensional representations of quantum analog of the enveloping algebra of a complex simple Lie algebra. Commun. Math. Phys. 117, 581-593 (1988)

[R2] Rosso, M.: Algèbres enveloppantes quantifiées, groupes quantiques compacts de matrices et calcul différential non commutatif. Duke Math. J. 61, 11-40 (1990)

[R3] Rosso, M.: Analogues de la forme de Killing et du théorème d'Harish-Chandra pour les groupes quantique. Ann. Sci. Éc. Norm. Sup 23, 445-467 (1990)

[S] Sweedler, M.: Hopf algebras. New York, Benjamin, 1969

[RT] Reshetikhin, N.Y., Turaev, V.G.: Invariants of 3-manifolds via link polynomials and quantum groups. Invent. Math. 103, 557-597 (1991)

[VD] Van Daele, A.: Quasi-discrete locally compact groups. Preprint (1993)

[V] Varadarajan V.S.: Lie groups, Lie algebras, and their representation. New York: Prentice Hall, 1974

[We1] Wenzl, H.: Hecke algebras of type $A_{n}$ and subfactors. Invent. Math. 92 345-383 (1988)

[We2] Wenzl, H.: Quantum groups and subfactors of type B, C and D. Commun. Math. Phys. 133, 383-433 (1990)

[We3] Wenzl, H.: Braids and invariants of 3-manifold. Invent. Math. 114, 235-275 (1993)

[Wo1] Woronowicz, S.L.: Compact matrix pseudogroup. Commun. Math. Phys. 111, 613-665 (1987)

[Wo2] Woronowicz, S.L.: Tannaka-Krein duality for compact matrix pseudogroups. Twisted SU(N) groups. Invent. Math. 93, 35-76 (1988)

[Ya1] Yamagami, S.: A note on Ocneanu's approach to Jones index theory. Int. J. Math. 4, 859-871 (1993)

[Ya2] Yamagami, S.: Modular theory for bimodules. J. Funct. Analysis, to appear

[Ya3] Yamagami, S.: Frobenius reciprocity in tensor categories. Preprint (1994)

[Ya4] Yamagami, S.: Crossed products in bimodules. Preprint (1993)

[Ya5] Yamagami, S.: On Ocneanu's characterization of crossed products. Preprint (1993) 
